\title{
Numerical and experimental study of residual stresses in a linear friction welded $\mathrm{Al}-\mathrm{SiC}_{\mathrm{p}}$ composite
}

\author{
Tea-Sung Jun ${ }^{1, a}$, Xu Song ${ }^{1, b}$, Fabio Rotundo ${ }^{2, c}$, Lorella Ceschini ${ }^{3, d}$, Alessandro \\ Morri $^{3, e}$, Philip Threadgill ${ }^{4, f}$, Alexander M. Korsunsky ${ }^{1,9}$ \\ ${ }^{1}$ Dept of Engineering Science, University of Oxford, Parks Road, Oxford OX1 3PJ, England \\ ${ }^{2}$ DIEM, University of Bologna, V. le Risorgimento 2, I-40136 Bologna, Italy \\ ${ }^{3}$ SMETEC, University of Bologna, V. le Risorgimento 4, I-40136 Bologna, Italy \\ ${ }^{4}$ TWI Ltd, Granta Park, Great Abington, Cambridge, CB21 6AL, UK \\ atea.jun@eng.ox.ac.uk, ${ }^{b} x u . s o n g @ e n g . o x . a c . u k,{ }^{c}$ fabio.rotundo@unibo.it, \\ dceschini@bomet.fci.unibo.it, ealessandro.morri@bomet.fci.unibo.it, ${ }^{f}$ philip.threadgill@twi.co.uk, \\ galexander.korsunsky@eng.ox.ac.uk
}

\begin{abstract}
Keywords: Linear Friction Welding, Residual Stresses, Neutron Diffraction, Process Modelling, Eigenstrain Reconstruction
\end{abstract}

\begin{abstract}
In the present study, the process modelling of AMCs linear friction weldment was carried out. Four major stages of the process (Part 1: Warm-Up; Part 2: Osci-Forging; Part 3: Forging; Part 4: Cool-Down) were identified and simulated consecutively to generate the temperature field and residual strains distribution within the model. An eigenstrain model calibrated by the neutron diffraction results was also employed to capture the permanent deformation distribution. Good agreement between the process modelling and the experimental measurements was found.
\end{abstract}

\section{Introduction}

Metal Matrix Composites (MMCs) are a class of materials in which a reinforcement phase is dispersed in a continuous metallic matrix. Over the past few decades, significant efforts have been devoted to the development of MMCs with improved physical and mechanical properties compared to the monolithic metals. The synergetic interaction between matrix, usually of light alloys (Al, Ti or $\mathrm{Mg}$ ) and the reinforcement, usually ceramic (oxides, carbides, nitrides) particles or fibres, bestows MMCs with enhanced mechanical properties over the parent unreinforced alloy [1]. Among the MMCs, aluminium matrix composites (AMCs) form a class of attractive light-weight materials possessing a good combination of high stiffness and strength, particularly in terms of their ratio to material density. The incorporation of stiff and hard ceramic reinforcement in the form of fibres or particles leads to a significant increase in the overall elastic modulus. Strength is also improved due to a variety of mechanisms, including grain refinement, creation of additional obstacles to dislocation movement, etc. Despite these advantages over conventional alloys, a significant limitation to the industrial application of AMCs is associated with the problems arising in conventional joining techniques, such as solute segregation in the alloy, and the degradation of the reinforcement phase.

Recently, linear friction welding has been successfully applied to join AMC materials, since the method avoids melting and solidification that cause the defects listed above [2]. Linear friction welding is a solid state joining process in which the bonding of two flat-edged components is completed by their relative reciprocating motion under axial (compressive) forces. In the course of the process significant heat is generated by friction at the component interface, resulting in continued displacement of plastically deformed materials [3-6]. Complex interaction between thermal and mechanical effects within the component makes understanding and optimising the process a significant technological challenge. In the literature, the LFW process for Ti alloys has been studied, with four distinct phases identified in terms of the applied shear force and displacement history [3-4]. However, for AMCs the process could not be elucidated sufficiently using the same classification. 
To improve the understanding of LFW process, different approaches to residual stress analysis have been used, including neutron diffraction, process simulation and eigenstrain reconstruction.

\section{Neutron Diffraction Measurement}

Linear friction welding of aluminium alloy matrix composite AMC225xe samples was performed at TWI (The Welding Institute, Cambridge, UK). AMC225xe is a high quality aerospace grade aluminium alloy reinforced with $25 \%$ by volume of ultra-fine particles of silicon carbide, manufactured by Aerospace Metal Composites Ltd (Farnborough, UK). The specification of the weldment can be seen in Figure 1 and the details of the material can be found in [2].

Residual strain measurement was carried out by neutron diffraction on the EXGIN-X instrument (ISIS, Rutherford Appleton Laboratory, UK). A heavy metal target is bombarded with pulses of highly energetic protons from a powerful accelerator, driving neutrons from the nuclei of the target atom. This leads to an extremely intense neutron pulse, delivered with only modest heat production in the neutron target. Figure 2 shows the illustration of the experimental setup. The diffraction patterns were collected as time-of-flight data by two detectors mounted perpendicularly to the incident beam. Due to the lower interaction with matter of neutrons in comparison with X-rays, the absorption of the beam is low, allowing deeper penetration in bulk samples whilst requiring larger gauge volume and longer counting time due to limited particle flux. Gauge volume size of $2 \times 2 \times 10 \mathrm{~mm}^{3}$ (elongated in the $z$ direction) and the counting time of about 30 minutes for each point were adopted. Steps of $1 \mathrm{~mm}$ were used near the bond line, providing overlapping and thus allowing more precision in the strain profile reconstruction. Increasing steps were used to measure strains far from the bond line.

The data analysis was carried out on the entire diffraction spectra obtained at each measurement position, using the Open Genie software for the display and analysis of data from the neutron scattering instruments at the ISIS facility [7]. The results from the multiple peak fitting analysis are converted into lattice d-spacing by combining the de Broglie's equation and Bragg's equation:

$$
d_{h k l}=\frac{h t}{\left(2 m L \sin \theta_{B}\right)},
$$

where $2 \theta_{B}$ is the neutron scattering angle, $d_{h k l}$ is the lattice spacing between the family of planes with $h k l$ Miller indices, $h$ Planck's constant, $m$ neutron mass, $L$ the flight path and $t$ the time-of-flight of the neutron. The elastic strain is determined from the change in the atomic interplanar distances $d_{h k l}$, as compared with the value $d_{h k l}^{0}$ measured in the unstressed (or reference) condition, $\varepsilon_{h k l}=\left(d_{h k l}-d_{h k l}^{0}\right) / d_{h k l}^{0}$ [8]. Due to the absence of reference strain-free samples, the unstrained lattice parameters $d_{h k l}^{0}$ were obtained from the pattern collected by placing the beam at the very corner of the plate, which was assumed to carry little or no residual macroscopic strain. A different reference was used for each half of the component, due to the fact that different parts may have come from slightly different production routes. The reference at the central point $(x=0)$ was taken to be the average value of the two far-field references.

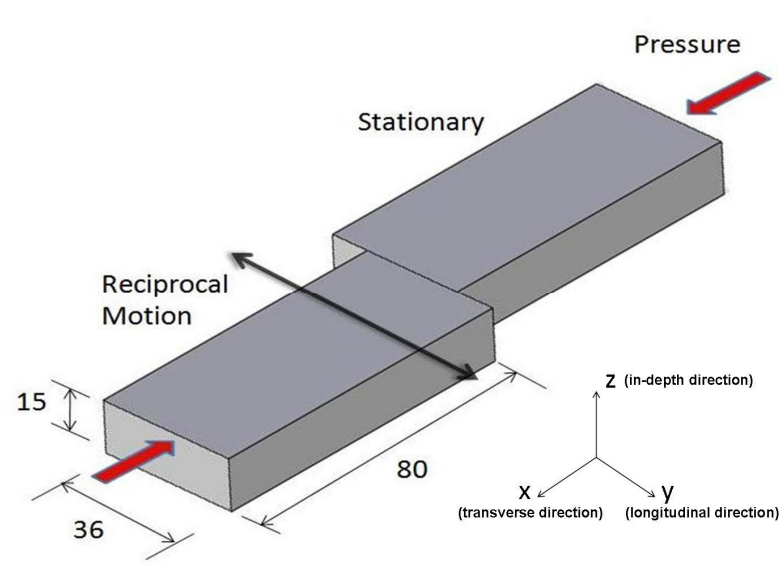

Fig. 1. Schematic of the AMCs linear friction welds

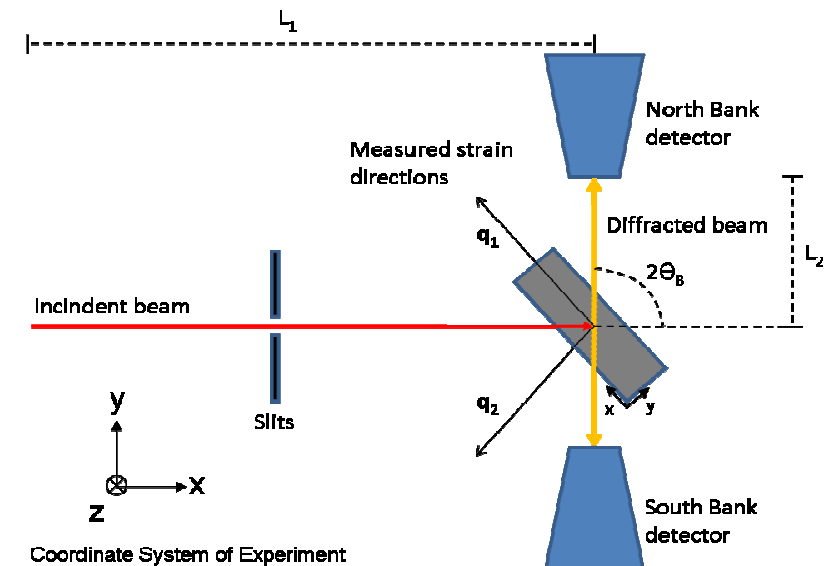

Fig. 2. Specification of the ENGIN-X diffractometer at ISIS 
Figure 3 shows the residual elastic $y$-strain distribution as a function of the $x$ position across the bond line. The $y$-strain exhibits a large tensile region in the vicinity of the weld zone as a consequence of the plastic deformation and cooling process involving flash extrusion of material, turning to residual compression further away from the bond line.

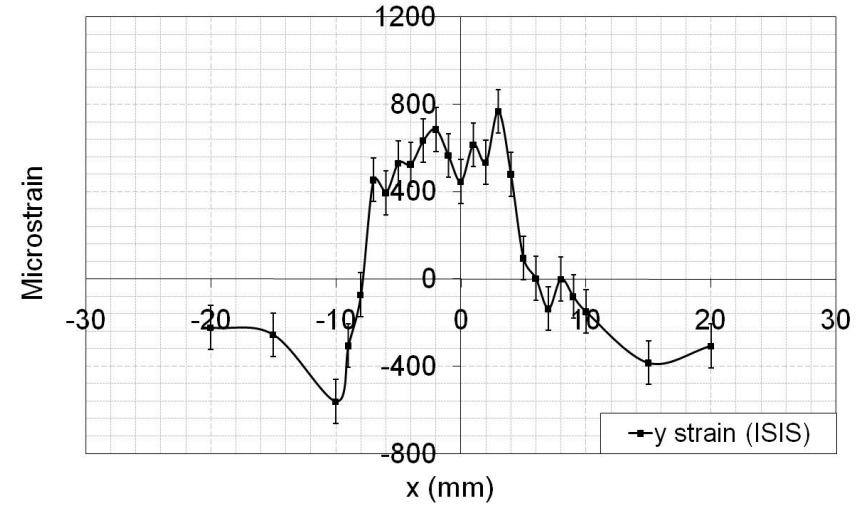

Fig. 3. Residual elastic $y$-strain distribution in the welds as a function of $\mathrm{x}$-axis position

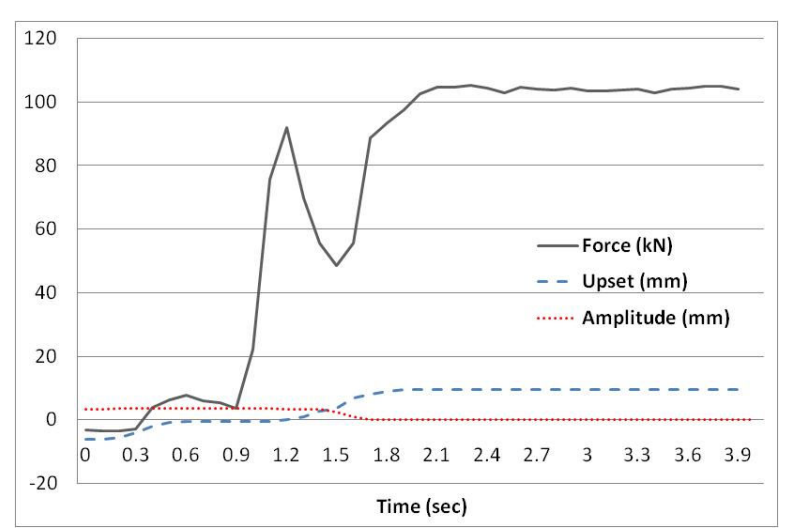

Fig. 4. The variation of the weld parameters with time

\section{Process simulation and the analysis of resulting residual stresses}

The LFW process can be divided into four distinct stages within the process timescale with. Four key representative parameters describe the process: amplitude of oscillation, oscillation frequency, axial force and burn-off. In the present study, the AMC225xe/AMC225xe LFW was performed at the amplitude of $2 \mathrm{~mm}$, frequency of $50 \mathrm{~Hz}$, axial compressive force of $100 \mathrm{kN}$ and burn-off of $2 \mathrm{~mm}$. The time variation of these parameters during the process can be found in Figure 4. The schematic diagram of the process is shown in Figure 5 and the characteristics of each stage are as follows:

\section{Stage $1(0.0-1.0 \mathrm{sec})$ : Warm-Up}

The two parts are aligned in the machine. Oscillating motion begins of part A whilst part B is maintained static, and then the two components are brought together. Once in contact, sufficient heat is generated by the solid friction due to the reciprocal motion under low force (or pressure). The wear and thermal softening leads to an increase of the real contact area and of the friction coefficient. It is of crucial to generate enough heat to attain plastification of the interface. For linear fiction welding to be possible, the process must overcome the heat loss via conduction to the parent material and radiation and convection to the atmosphere. No weld penetration (upset) is experienced in this part.

\section{Stage $2(1.0-1.5 \mathrm{sec})$ : Osci-Forging}

In this stage, whilst part A maintains the same oscillatory motion with respect to part B as in stage 1, the axial force is applied to push the two components together. The force is increased steeply to about $90 \mathrm{kN}$ until 1.2 seconds, when an abrupt drop in the force occurs in the following 0.3 seconds. This phenomenon is related to the setting in of the burn-off process. Once axial shortening begins, the increasing force starts to decrease until the $2 \mathrm{~mm}$ burn-off is accomplished. Throughout the process, interface rubbing continues to remove the oxide layer and to reveal fresh surfaces suitable for bonding, also creating an extrusion known as "flash" of different shape depending on the material(s) being bonded and extruded. The actual final shortening exceeds slightly the initial setting due to inertia. Oscillatory motion is ramped down when the axial shorting reaches the set burn-off. With no further heat generation, nor burn-off, the heated zone expands into the bulk of the material, leading to yield stress reduction and encouraging plastic flow and permanent inelastic deformation.

\section{Stage $3(1.5-2.0 \mathrm{sec})$ : Forging}

This stage resembles conventional hot forging process in some respects. The force increases up to about $100 \mathrm{kN}$ whilst two parts continue to be pressed against each other. As a result, large amount of "flash" of plastically deformed material is expelled from the bond line resulting in the consolidation 
of the weld and further axial shortening, which increases approximately linearly with time, till about 2 seconds. Afterwards no further axial shortening can be found unless force is increased.

\section{Stage 4 (2.0-4.0 sec): Cool-Down}

While the compressive force continues to be maintained, the newly joined piece is cooled down to room temperature. Once the process is complete the total upset was found to be $9.37 \mathrm{~mm}$, the total length of welds thus being changed from $160 \mathrm{~mm}$ to $150.63 \mathrm{~mm}$.

Part 1: Warm-Up

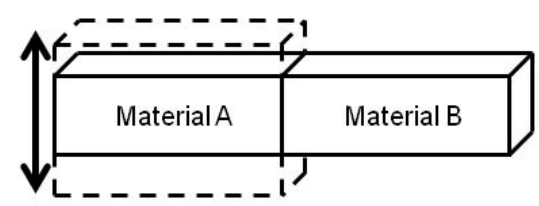

Part 3: Forging

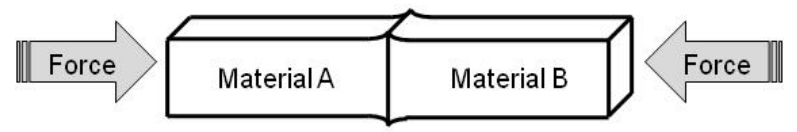

Part 2: Osci-Forging

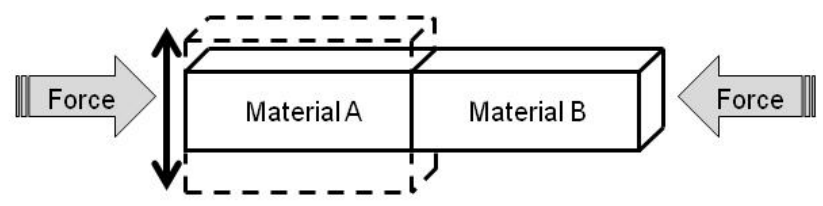

Part 4: Cool-Down

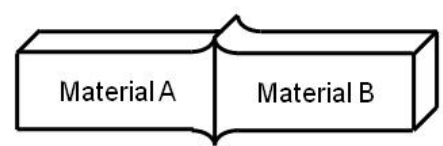

Fig. 5. Schematic of the linear friction welding process

The thermal and mechanical properties of the AMC225xe material were obtained from the company technical data sheet [9]. It was assumed that the plastic behaviour follows linear kinematic hardening, allowing reverse yielding. The heat conduction coefficient was assumed to be equal to $100 \mathrm{~W} /\left(\mathrm{m}^{2} \mathrm{~K}\right)$. The one eighth-geometry of the component was represented by a 3D block with half of the original width and height, and half length of the full assembly. Due to symmetry, the counterpart component was represented by a rigid surface. The model set-up is illustrated in Figure 6 with the contours of the residual von Mises stress distribution displayed at the end of the process.

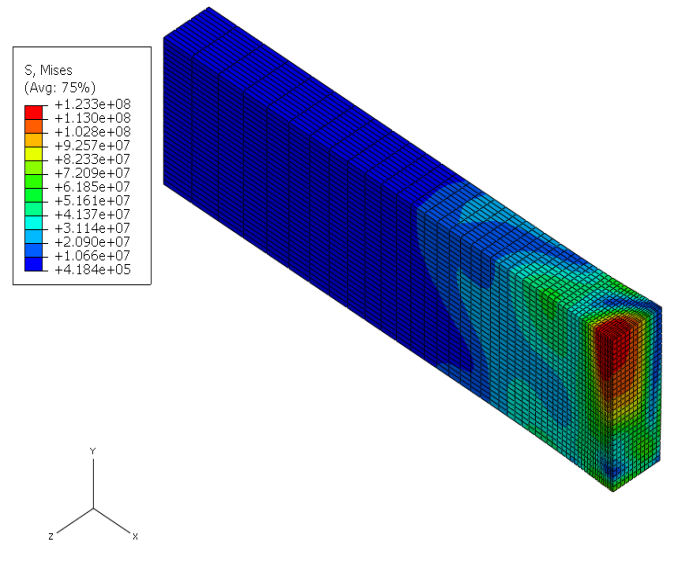

Fig.6. The 3D set-up for the LFW process simulation and its resulting $\mathrm{RS}$ profile at the end stage

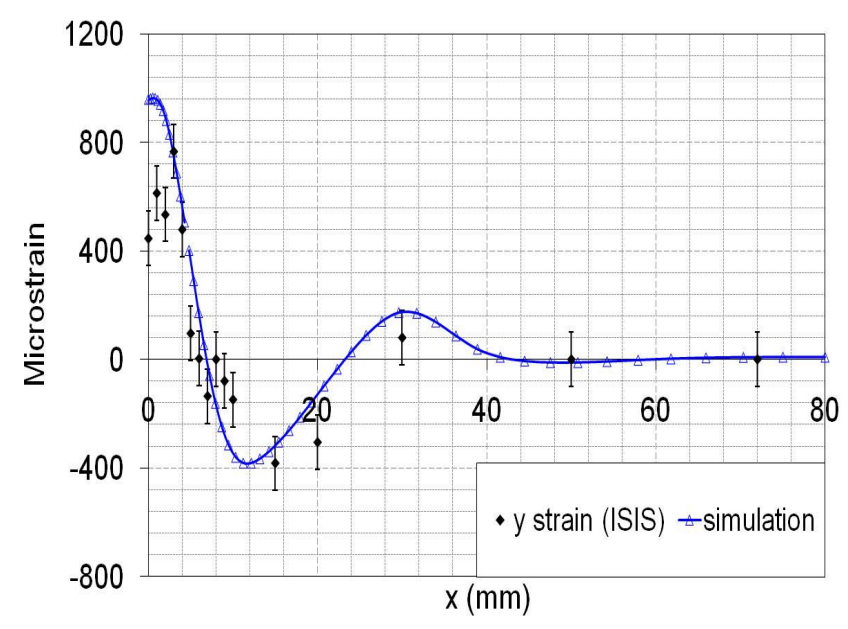

Fig.7. Residual elastic y-strain distributions via experiment and process modelling simulation

The simulation of linear friction welding followed the process stages identified above. The mutual influence of thermal and deformation effects is apparent, with heat transfer affecting stress field generation. Steps of the simulation coincided with the four stages of the process. Displacement control was used in the simulation, since, as seen in Figure 4, it is smooth and readily prescribed, unlike the spiky force-time plot not suitable for incremental control. In the simulation of the Warm-Up and Osci-Forging stages, oscillation of the component is replaced by surface heat flux. 
Although this clearly is a simplification, it was used to test the hypothesis that the main function of the reciprocal movement is to evolve interface heat. The average heat generation rate is given as [4]:

$$
q_{0}=\frac{2}{\pi} \mu P_{N} \alpha \omega
$$

where $q_{0}$ is the heat rate, $P_{N}$ is the normal pressure applied on the component, $\mu$ is the friction coefficient assumed to be equal to 0.5 [10], $\alpha$ is the oscillation amplitude and $\omega$ is the oscillation frequency. Other process parameters were set to the values measured during welding.

At the end of Cool-Down stage, the residual $y$-strain profile along the $x$-axis is extracted in accordance with the neutron experiment diffraction gauge volume, which is found from the simulation by averaging over multiple nodal points along a path in the $x$ direction (Figure 7 ). The simulation result clearly captures the trend and the magnitude of the residual strain profile within the experimental tolerance. The minor discrepancy arises close to bond line region where the FE prediction gives an over-estimate. The discrepancy may be caused by our lack of accounting for the local effect of high interface shear stress on local plastic flow, and the possibility of reverse yielding.

\section{Inverse Eigenstrain Analysis}

Welding gives rise to permanent inelastic strain (eigenstrain) $\varepsilon^{*}$ that is a combination of plastic deformation and thermal expansion mismatch between parts [11]. In the small strain approximation, additive decomposition of total strain is given as the sum of elastic strain and eigenstrain,

$$
\varepsilon_{\text {total }}=\varepsilon_{e}+\varepsilon^{*}
$$

where $\varepsilon_{e}$ is elastic strain, and eigenstrain $\varepsilon^{*}=\varepsilon_{p l}+\varepsilon_{t h}$, with $\varepsilon_{p l}$ the plastic strain and $\varepsilon_{t h}$ the thermal strain. Distributions of eigenstrains embedded in the workpiece are responsible for the residual stresses observed experimentally. Saint-Venant strain compatibility equations and elastic equilibration analysis show that residual stresses arise in a solid body if and only if an incompatible distribution of eigenstrains is present [12-13]. Both conceptually and mathematically it is convenient to consider eigenstrain as the source of residual stresses. Using a known eigenstrain field to deduce the residual elastic strain (and stress) field is the direct problem of eigenstrain analysis. The inverse problem of eigenstrain analysis concerns using the knowledge of residual elastic strains (or stresses) at a number of measurement points to retrieve the underlying source eigenstrain field.

Frameworks for inverse eigenstrain analysis proposed in [14-15] make use of a robust and efficient least squares approach to find the unknown eigenstrain distributions from residual elastic strains measured at a finite number of experimental points [16-17]. In the present work, a 2D plane stress FE model was set up reflecting the geometry of the experiment. Since the plastic strain distribution is unknown a priori, the inverse method must be used to extract the inelastic deformation distribution by matching the experimental residual elastic strain data. Unknown eigenstrain distribution is represented by a linear superposition of overlapping triangular pulse basis functions, $\xi_{i}(x)$, known as the simtri method [18], so that

$$
\varepsilon^{*}(x)=\sum_{i=1}^{N} c_{i} \xi_{i}(x)
$$

where $c_{i}$ denote the unknown coefficients. For the eigenstrain given by the sum in equation (4), the resulting residual stresses and residual elastic strains are also given by the sum, with appropriate coefficients $c_{i}$, of the readily obtainable FE individual solutions $\Xi_{i}$ for each basis function $\xi_{i}(x)$.

The unknown coefficients can then be found by least squares matching between the prediction and the residual elastic strains measured experimentally, following the procedure described in detail in the literature [17]. Figure 8 shows that the elastic strains deduced by inverse eigenstrain simtri method capture the trend and magnitude of residual strain profile within the experimental tolerance. 


\section{Conclusion}

The study described in this paper combined several different approaches to analyse residual strains in linear friction weldments of AMCs, namely, neutron TOF diffraction to measure strains, process modelling simulation, and inverse eigenstrain analysis. Adequate agreement was obtained between different techniques, suggesting that the choice of methods was justified. However, further improvements to the process modelling capability are required. Firstly, it is necessary to capture the details of the residual elastic strain variation close to the bond line. Secondly, dependence of the residual strain profile on process parameters and material properties needs to be investigated. For example, the consideration of LFW joints between AMC and unreinforced Al alloy is likely to provide interesting insight into the effect of material property on the LFW process. Finally, more detailed 3D maps of residual strain states are required for better validation of the process model.

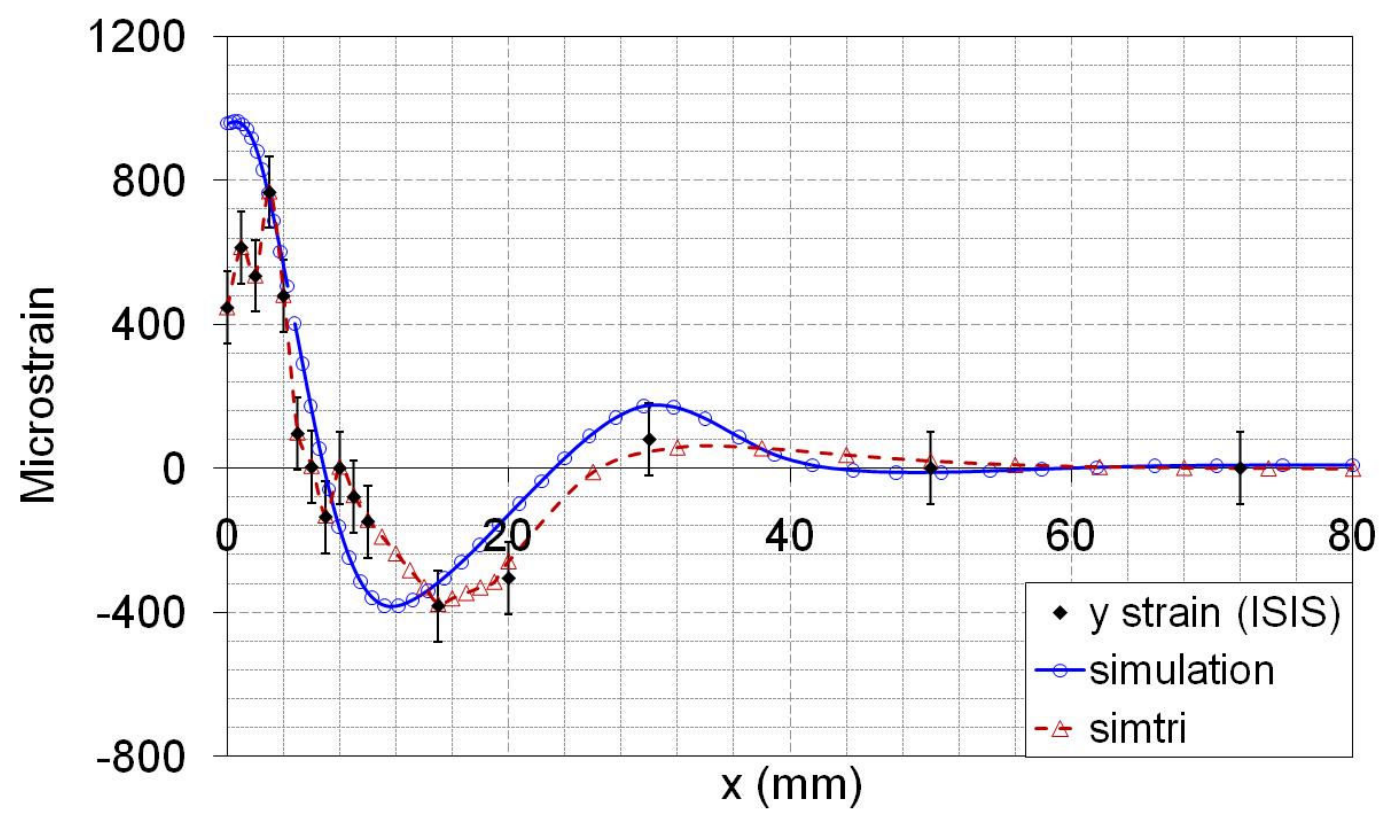

Fig. 8. Residual elastic y-strain distributions via experiment, process modelling simulation, and simtri method

\section{References}

[1] T.W. Clyne and P.J. Withers: An Introduction to Metal Matrix Composites (CUP, UK 1993).

[2] T-S. Jun, F. Rotundo. L. Ceschini and A.M. Korsunsky: Key Eng. Mater. Vol.385-387 (2008), p. 517-520

[3] A. Vairis and M. Frost: Wear 217 (1998) p. 117-131

[4] A. Vairis and M. Frost: Mater. Sci. Eng. A 292 (2000) p. 8-17

[5] P. Wanjara and M. Jahazi: Metallurgical and Material Transactions A 36A (2005) p. 2149-2164

[6] M.R. Daymond and N.W. Bonner: Physica B 325 (2003) p. 130-137

[7] F.A. Akeroyd, R.L. Ashworth, S.D. Johnston, J.M. Martin, C.M. Moreton-Smith and D.S. Sivia: OPEN GENIE User Manual

[8] J.R. Santisteban, M.R. Daymond, J.A. James and L. Edwards: J. Appl. Cryst. 39 (2006) p.812-825

[9] Aerospace Metal Composites Limited: Particle Reinforced Aluminium Alloy AMC225xe Technical Data Sheet (2006) p.1-3

[10] B. Venkataraman and G. Sundararajan: Acta Mater. 44(2) (1996) p.451-460

[11] T. Mura: Micromechanics of defects in solids (Martinus Nijhoff, Boston 1987)

[12] A.M. Korsunsky: J. Mechanics Matls. Structs. 1 (2006) p.101-119

[13] A.M. Korsunsky: J. Strain Analysis 44 (2009) p.29-43

[14] Y. Ueda, K. Fukuda and Y.C. Kim: Transactions of the ASME 108 (1986) p.328-334

[15] T.L. Panontin, M.R. Hill: Int. J. of Fracture 82 (1996) p.317-333 
[16] A.M. Korsunsky: J. Strain Analysis 41(3) (2006) p.195-204

[17] A.M. Korsunsky, G.M. Regino, D. Nowell: Int. J. Solids and Structures 44 (2007) p.4574-4591

[18] X. Song, S. Chardonnet, G. Savini, S.Y. Zhang, W.J.J. Vorster, A.M. Korsunsky: Matls. Sci. Forum 571-572 (2008) p.277-282 